\title{
Corneal damage and its recovery after instillation of preservative-free versus preserved latanoprost eye drops
}

Daisuke Inoue $^{\mathrm{a}}$, Yasser Helmy Mohamed ${ }^{\text {ab* }}$, Masafumi Uematsu ${ }^{\mathrm{a}}$, and Takashi Kitaoka ${ }^{\text {a }}$

${ }^{a}$ Department of Ophthalmology and Visual Sciences, Graduate School of Biomedical Sciences, Nagasaki University, 1-7-1 Sakamoto, Nagasaki, Nagasaki 852-8501, Japan;

${ }^{b}$ Department of Ophthalmology, EL-Minia University Hospital, EL-Minia, Egypt *corresponding author: Yasser Helmy Mohamed

Department of Ophthalmology and Visual Sciences, Graduate School of Biomedical Sciences, Nagasaki University, 1-7-1 Sakamoto, Nagasaki, Nagasaki 852-8501, Japan Tel: +81-95-819-7345; Fax: +81-95-819-7347; E-mail: yasserhelmy@nagasaki-u.ac.jp Word Count: 4,516 words 


\title{
Corneal damage and its recovery after instillation of preservative-free versus preserved latanoprost eye drops
}

\begin{abstract}
Purpose: Latanoprost ophthalmic solution is highly effective as a therapeutic agent for glaucoma and is applied worldwide. However, harmful effects on the corneal surface have been reported regarding the commercially available latanoprost ophthalmic solution. Corneal surface toxicity may be caused by the added preservative of the ophthalmic solution. In order to ascertain whether latanoprost itself can damage the cornea or if this is solely due to the added preservatives, this study attempted to determine the corneal changes that occur at different time periods following usage of preservative-free versus preserved latanoprost eye drops.
\end{abstract}

Materials and Methods: Preservative-free latanoprost eye drops (Monoprost ${ }^{\circledR}$ ) or preserved latanoprost eye drops (Xalatan ${ }^{\circledR}$ ) containing $0.02 \%$ benzalkonium chloride (BAC) were instilled in the corneas of rabbits. For each of the two different eye drop solutions, the rabbits used in this experiment were divided into three exposure groups: the 1 minute, 24 hour, and 1 week groups. Corneal transepithelial electrical resistance (TER) and scanning electron microscopy (SEM) were examined immediately (1 minute) after instillation, at 24 hours after instillation, and at 24 hours after 1 week of daily instillations of latanoprost. Hank's balanced salt solution was used in the control group.

Results: The mean corneal TER of the control group was $933.8 \pm 279.0 \Omega \mathrm{cm}^{2}$. In preservative-free latanoprost instilled corneas, there was no significant decrease in the TER or morphological changes at any of the time points, with the relative TER values of $117 \pm 38 \%, 100 \pm 34 \%$, and $93 \pm 21 \%$ for the 1 minute, 1 day, and 1 week time points, respectively. In preserved latanoprost instilled corneas, SEM showed that only the immediate group exhibited superficial cell damage and a significant decrease in the corneal TER compared to the controls and other time points and to the immediate preservative-free latanoprost corneas. 
In the preserved latanoprost groups, the relative TER values were $18 \pm 5 \%, 110 \pm$ $28 \%$, and $92 \pm 10 \%$, for the three respective observation time points.

Conclusions: Preservative-free latanoprost can be safely instilled to the corneal epithelium. Latanoprost with $0.02 \% \mathrm{BAC}$ has an immediate deleterious impact on the corneal epithelium; however, it disappears within 24 hours after instillation.

Keywords: glaucoma medications; electron microscopy; electrical resistance; cornea; latanoprost; preservative; benzalkonium chloride

\section{Introduction}

Glaucoma is one of the commonest causes of irreversible visual impairment and blindness. Anti-glaucomatous therapy aims to prevent progressive optic nerve damage, usually by lowering intraocular pressure (IOP). Prostaglandin (PG) analogues have become first-line treatments among glaucoma medications. Among these PGs, latanoprost provides a highly advantageous balance in terms of IOP lowering efficacy and tolerance, thus remaining the most frequently used PG analogue worldwide [1]. However, the use of a commercial solution of a preserved latanoprost formulation has been proposed, raising a number of issues, especially in patients with an abnormal or sensitive ocular surface.

The most widely used preservative is benzalkonium chloride (BAC). The harmful effects of BAC on conjunctival and corneal epithelial cell layers have been shown in numerous clinical and experimental studies [2,3]. Several in vivo animal studies and ex vivo studies using human tissue-derived cell lines have also demonstrated the detrimental effects of $\mathrm{BAC}[3,4]$. In addition, the ocular surface side effects of antiglaucoma medications should also not be neglected, as they may deeply impact a patient's quality of life, compliance, or later surgical outcome. Reducing preservative 
exposure potentially lessens adverse events, which could lead to improved tolerability, a lower percentage of treatment discontinuations, and higher adherence in patients treated with antiglaucoma medications [5]. Preservative-free (PF) latanoprost (Monoprost ${ }^{\circledR}$ ) is a formulation of latanoprost that has been approved for use in the European Union in patients with primary open-angle glaucoma (POAG)/ocular hypertension [6].

Pauly et al. [7] compared the preserved and PF latanoprost effects in vitro and in vivo, on the human reconstituted corneal epithelial SkinEthics model, and using an acute rabbit toxicological model, respectively. Their study showed that while there was no significant difference compared with the control, there was a significant difference with preserved latanoprost. However, their in vivo studies that used slit lamp biomicroscopy examination, conjunctival impression cytology (CIC), and corneal in vivo confocal microscopy (IVCM), did not utilize electrophysiological techniques. To the best of our knowledge, our study is the first to compare PF (Monoprost ${ }^{\circledR}$ ) and preserved latanoprost $\left(\right.$ Xalatan $\left.{ }^{\circledR}\right)$ eye drops with regard to transepithelial electric resistance (TER) measurements and scanning electron microscopy (SEM) evaluations. In addition, the test solution used in this study was instilled 15 times in the animal's eye at 5-minute intervals. In order for the evaluation to be conducted under conditions closer to actual clinical use, our study evaluated the effects that occur following instillation at 24 hour intervals as described later.

Measuring corneal TER is a suitable method for quantitative and continuous evaluation of corneal permeability and irritancy. TER reflects the barrier function of the epithelium. A lower corneal TER indicates that more electrical current is able to penetrate through the damaged superficial cells and the tight junctions between them. In addition, it has been reported as a very sensitive test for measuring the electrical properties of the cornea [8]. We previously developed a method of measuring the TER 
of live rabbit corneas [9]. After developing this in vivo method of measuring the TER of rabbit corneas, we demonstrated that BAC concentrations between $0.005 \%$ and $0.02 \%$ immediately caused acute corneal barrier dysfunction [10-12].

In addition, we have confirmed the acute detrimental effect of latanoprost with BAC (Xalatan $\left.{ }^{\circledR}\right)$ on the corneal epithelium. In that previous study, we proved that latanoprost with BAC caused an acute decrease in the TER measurement of the corneal epithelium within 1 minute following instillation of the drug [13]. In this study, we used TER measurements and SEM to examine the corneal epithelial changes and the recovery within 24 hours after the usage of PF versus preserved latanoprost eye drops. We used an in vivo experiment in this study to evaluate the regenerative power.

\section{Materials and Methods}

\section{Chemicals}

$\mathrm{Ca}^{2+}$ and $\mathrm{Mg}^{2+}$ free Hank's Balanced Salt Solution (HBSS) was obtained from Invitrogen Corp. (Carlsbad, CA, USA). Commercially available Monoprost ${ }^{\circledR}$ containing PF latanoprost in a single-dose container (Thea Pharmaceuticals Ltd., Clermont-Ferrand, France) and Xalatan ${ }^{\circledR}$ containing latanoprost with $0.02 \%$ BAC in a multi-dose bottle (Pfizer, New York, NY, USA) were used in this study.

\section{Experimental Animals}

Male white Japanese rabbits (KBT Oriental, Tosu, Japan) weighing 2.5-3.0 kg were individually housed in cages under a controlled temperature $\left(21^{\circ} \mathrm{C}\right)$, humidity $(50 \pm$ 5\%), and a 12/12-hour light/dark cycle at the Laboratory Animal Center for Biomedical Research, Nagasaki University School of Medicine. The study was initiated once the rabbits reached weights of $3.0-4.0 \mathrm{~kg}$, as this was the point where the corneal diameters 
were of suitable size for experimentation. All rabbits were treated in compliance with the ARVO Statement for the Use of Animals in Ophthalmic and Vision Research.

\section{Latanoprost Instillation and Times of Examinations}

Animals were divided into 6 experimental and 1 control groups. PF latanoprost eye drops (Monoprost ${ }^{\circledR}$ ) were instilled in groups 1, 2, and 3, while preserved latanoprost

eye drops (Xalatan $\left.{ }^{\circledR}\right)$ were instilled in groups 4,5 , and 6. Corneal TER and SEM were examined immediately ( 1 minute) following instillation, 24 hours after instillation, and 24 hours after the last instillation following a daily application that was given over a 1 week period (groups 1, 4), (groups 2, 5), and (groups 3,6) respectively. The animals in the control group were administered HBSS.

\section{Corneal TER MeasurementsIn Vivo}

Rabbits were anesthetized using an intramuscular injection of $30 \mathrm{mg} / \mathrm{kg}$ ketamine (Ketalar, Daiichi Sankyo, Tokyo, Japan) and 5 mg/kg xylazine (Celactal, Bayer Yakuhin, Osaka, Japan). A $1.0 \mathrm{~mm}$ diameter custom-made $\mathrm{Ag} / \mathrm{AgCl}$ electrode (Physiotech, Tokyo, Japan) was inserted into the anterior chamber, following a small incision made in the peripheral cornea using an 18-gauge sharp needle (Terumo, Tokyo, Japan). A $6.0 \mathrm{~mm}$ internal diameter $\left(0.28 \mathrm{~cm}^{2}\right.$ inner area) nitrile rubber O-ring (Union Packing, SAN-EI, Osaka, Japan) was fixed on the cornea using biomedical adhesive (Alon-Alpha A, Daiichi Sankyo, Tokyo, Japan). Subsequently, $80 \mu \mathrm{L}$ of HBSS were added in the ring, with a second electrode then placed in the HBSS on the cornea. The TER was measured in real time using a volt-ohm meter (EVOMX, World Precision Instruments, Sarasota, FL, USA) generating a $\pm 20 \mu \mathrm{A}$ AC square wave current at 12.5 Hz. The specific methodology and photographs of the in vivo corneal TER measurement system have been previously published [9-12]. The sample size for the corneal TER 
study was set at 4 to 8 , which we found to be sufficient for our statistical analyses in our previous TER studies [9-12].

\section{SEM Study}

Rabbits were anesthetized with an intramuscular injection of $30 \mathrm{mg} / \mathrm{kg}$ ketamine and 5 $\mathrm{mg} / \mathrm{kg}$ xylazine. They were then sacrificed using a lethal dose of intravenous sodium pentobarbital (Nembutal, Dainippon Pharmaceutical, Osaka, Japan) and prepared for SEM examinations. The corneas were carefully excised, fixed in $4 \%$ glutaraldehyde in $0.05 \mathrm{M}$ cacodylate buffer for 1 hour, and then post-fixed in $1 \%$ osmium tetroxide in veronal acetate buffer containing $0.22 \mathrm{M}$ sucrose. The fixed materials were dehydrated through a series of ethanol washes. Corneas were placed in $t$-butyl alcohol, treated in a freeze-drying apparatus (EIKO ID-2, EIKO, Tokyo, Japan), and then sputter-coated with gold using an auto fine coater (JEOL JFC-1600, JEOL, Tokyo, Japan). The surface of the corneal epithelium was observed by a scanning electron microscope following processing (Hitachi S2360, Hitachi, Ibaragi, Japan).

\section{Statistical Analysis}

All results are expressed as the mean \pm standard deviation of at least four experiments. Statistical comparisons were performed using an analysis of variance followed by Tukey's test for the relative TER among the same test solutions for the experimental groups and controls. An unpaired student's t-test was used to compare the two groups of different solutions at each of the time points. Values of $\mathrm{p}<0.01$ were considered to indicate statistical significance. 


\section{Results}

\section{Corneal TER Measurement of Latanoprost In Vivo}

The mean corneal TER for the live rabbits of the control group was $933.8 \pm 279.0 \Omega$ $\mathrm{cm}^{2}$. Figure 1 shows the relative TER value after corneal exposure to PF latanoprost and preserved latanoprost after different periods of time. In the PF latanoprost instillation groups, relative TER values were $117 \pm 38 \%, 100 \pm 34 \%$, and $93 \pm 21 \%$ for the 1 minute, 1 day, and 1 week time periods, respectively. No statistical difference among these groups was observed. In the preserved latanoprost instillation groups, the relative TER values were $18 \pm 5 \%, 110 \pm 28 \%$, and $92 \pm 10 \%$ for the 1 minute, 1 day, and 1 week time periods, respectively. The relative TER at 1 minute was significantly lower than that for the control, 1 day, and 1 week values $(\mathrm{p}<0.01)$. Also, at 1 minute, the relative TER was significantly lower in the preserved versus the PF latanoprost group $(\mathrm{p}<0.01)$.

[Figure 1 near here]

\section{Scanning Electron Microscopy Observation}

After instillation of HBSS in the superficial cells of the cornea of the control eyes, SEM showed a normal appearance with normal microvilli (Figure 2A). The superficial cells of the corneas exposed to PF latanoprost also exhibited a normal appearance with normal microvilli at all of the time points (Figure 2B, 2C, 2D). Immediately after the instillation of the preserved latanoprost in the cornea, SEM examination showed that there was damage to the superficial cells of the corneal epithelium, which exhibited lost or degenerated microvilli, a loss of adhesions with adjacent cells, and cracks appearing between the cells (Figure 3A). At 1 day and 1 week after instillation of the preserved 
latanoprost, the corneas appeared to be normal with normal microvilli (Figure 3B, 3C).

[Figure 2 near here]

[Figure 3 near here]

\section{Discussion}

Among the current ocular hypotensive medications employed in the treatment of openangle glaucoma and ocular hypertension, the first-line choice is topical application of PG analogues. This choice is based upon previous studies that have shown their efficacy in lowering IOP with few systemic side effects and the ease of the once daily dosing $[14,15]$. However, other clinical studies have shown that the topical application of PG analogues can cause ocular surface toxicity $[16,17]$. Furthermore, the toxicity of commercial PG analogues has often been associated with the use of BAC. BAC is one of the most commonly used preservatives because of its higher antimicrobial efficiency, stability, and low cost $[2,18]$. A huge number of animal and in vitro studies have been conducted for more than two decades using a considerable variety of models, cells, and tissues. These studies have demonstrated that BAC may cause or enhance harmful consequences on the eye structures of the anterior segment, including the tear film, cornea, conjunctiva, and even the trabecular meshwork [19]. Despite the consistent and solid data, along with the warnings from the results of observational surveys and individual case series, BAC is still used as the main preservative in eye drops, with very 
few alternatives having been developed [19].

We developed a method of measuring the TER of live rabbit corneas [9]. After developing an in vivo method for measuring the TER of rabbit corneas in our previous studies, we demonstrated that BAC concentrations between $0.005 \%$ and $0.02 \%$ immediately caused acute corneal barrier dysfunction [10-12]. We additionally used SEM to confirm the acute detrimental effect of latanoprost with BAC on the corneal epithelium. Furthermore, we proved that latanoprost with BAC caused a significantly acute decrease in the TER measurement of the corneal epithelium at 1 minute after the drug instillation [13].

Many methods have been used to evaluate corneal irritation and permeability induced by ophthalmic drugs. Drug toxicity must be rapidly evaluated because topically instilled drugs rapidly dilute with tears. Previously, we described a method for assessing acute corneal change after drug instillation by measuring the TER in vivo within seconds [9]. In general, TER reflects the barrier function of the epithelium, with lower corneal TER values indicative of the penetration of greater amounts of electrical current through the damaged superficial cells and tight junctions existing in the epithelium. TER is a sensitive, reliable, and versatile test of corneal epithelial barrier function, and thus is a useful indicator of corneal toxicity [9-12,20]. Our developed in vivo corneal TER measurement system uses custom-designed thin stick electrodes and a volt-ohm meter, which can measure the barrier function of intact corneas in rabbits. This design more accurately reflects the clinical instillation of ophthalmic drugs and gives us relevant data about the acute corneal toxicity of some eye drops [9-12].

Our study's results once again confirmed the detrimental effect of preserved latanoprost on the corneal epithelium immediately after application of the drug as well as the fact that the design of this study was consistent and reproducible. Surprisingly, 
the detrimental effect disappeared at 24 hours after application of the preserved latanoprost as well as at 1 week after daily latanoprost applications. We additionally confirmed that there were no significant differences between the TER measurements at 24 hours after application of the preserved latanoprost as well as at 1 week after daily latanoprost application in the control group. This shows that the regenerative capability of the corneal epithelium can reverse the immediate detrimental effect of the preserved latanoprost within 24 hours of instillation. We also confirmed that this regenerative power is a repetitive process that continued to occur each day during the week of daily exposures to preserved latanoprost. These findings were supported by the SEM results. Conversely, we determined no detrimental effect of PF latanoprost on the corneal epithelium at any of the different time periods that were examined (immediately, after 1 day, and after 1 week of daily application). These results were confirmed by both our TER measurements and the SEM evaluations. To the best of our knowledge, this is the first study to compare the PF (Monoprost ${ }^{\circledR}$ ) and preserved latanoprost $\left(\right.$ Xalatan $\left.{ }^{\circledR}\right)$ eye drops in regard to TER measurements and SEM evaluations. However, there are other previous studies that have compared the efficacy (IOP reduction) and safety of PF latanoprost to preserved latanoprost in ocular hypertension or POAG patients $[6,21,22]$. These studies concluded that PF latanoprost is equally efficient as the preserved formulation, having improved local tolerance. These statements, regarding the tolerance of the drug, can be supported by our current results. Liang et al. [23] concluded that PF tafluprost induced no obvious cytotoxicity, showed the least expression of inflammatory or apoptotic markers, and revealed preservation of the membrane immune-staining of tight junction proteins in contrast to the BAC-containing solutions. Pellenin et al. [24] also concluded that the cytotoxic effects of latanoprost, travoprost, and bimatoprost were due to the BAC concentration in 
their formulations, with PF tafluprost found to be the least toxic of all the drugs tested. In addition, Pauly et al. [7] demonstrated that preserved latanoprost decreased the cell viability of three-dimensional reconstituted human corneal epithelia at levels close to $50 \%$, while PF latanoprost slightly decreased the cell viability to $83 \%$. Furthermore, Pauly et al.[7]showed that there was a significant difference with preserved latanoprost, despite the fact that there was no significant difference compared with the control.

Most of the previous studies that have conducted comparisons between the two formulations have confirmed the acute toxic effect of preserved latanoprost on the corneal epithelium and the less toxic effect of PF prostaglandin analogues. Crichton et al. [17] concluded that despite preservative formulations, there were no significant differences in the objective clinical measures of the ocular surface tolerability after 3 months of treatment with bimatoprost (with $0.02 \% \mathrm{BAC}$ ), travoprost (with sofZia), and latanoprost (with $0.02 \%$ BAC). Also, our findings are supported by another study[25] that concluded that the reduced densities of the superficial epithelial cells in all of the glaucoma patient groups, with the exception for the PF group, were related to the toxic effects of BAC. The preservative groups also exhibited an increased density of basal epithelial cells, which may be attributable to a proliferate stimulus from the superficial layer that occurs in order to compensate for the superficial epithelial loss.

$\mathrm{BAC}$ is a quaternary ammonium compound that is most commonly used as a preservative in topical ophthalmic preparations. It is a highly effective antimicrobial agent that acts by denaturing proteins and disrupting cytoplasmic membranes [2]. Conversely, non-preserved eye drops could raise the risk of contamination. Moreover, PF artificial tears in re-closable containers have been shown to be at risk of contamination after multiple uses over 10 hours [26]. The risk was higher in older patients who had inappropriate finger manipulation, which may be the hallmark of 
patients with chronic eye diseases, namely glaucoma or dry eye disease [19]. Thus, the creation of a new PF latanoprost in a single dose container can protect patients from this type of contamination, which adds a greater safety level for long-term drug users.

Despite the number of articles supporting the deleterious effect of BAC on the ocular surface $[2,4,5,16,19]$, the debate continues on the benefit of BAC in eye drop formulations. An argument supporting BAC use arises from its capacity to increase corneal permeability, thereby acting as a penetration enhancer for active compounds and improving their pharmacologic effects [27,28]. Conversely, by Gross et al.'s prospective clinical study [29] demonstrated that BAC-free travoprost presented similar IOP lowering efficacy as BAC containing travoprost. Furthermore, a comparative pharmacokinetic study did not report finding any significant difference in the tafluprost concentration in rabbit aqueous humor between the BAC-associated and BAC-free formulations [30].

In addition, other studies have concluded that PF latanoprost has the same efficacy as the preserved formulation, with an improved local tolerance $[6,21,22]$. These previous studies contradict the assumption that BAC improves the pharmacologic effects of drugs containing the preservative.

There is evidence to support that BAC preserved medications are associated with a reduction of the tear film break-up time and basal tear secretion, a decrease of goblet cell density, the development of subconjunctival fibrosis, most likely related to an increase in inflammatory cells, the overexpression of HLA-DR, the intercellular adhesion molecule 1 (ICAM-1), IL-6, IL-8, or IL-10 in the epithelium, and increased apoptosis rates in the conjunctival epithelial cells [7,31]. Our study demonstrated that the regenerative power of the corneal epithelium can overcome the acute deleterious effect of preserved latanoprost exposure within 24 hours. However, a further studies are 
required in order to provide undisputed proof. Moreover, an additional study examining the effect of preserved latanoprost on the corneal epithelium over longer periods of time will also be needed.

Concluding, our results show that $\mathrm{PF}$ latanoprost (Monoprost $\left.{ }^{\circledR}\right)$ is safe regarding corneal epithelium application. In contrast, preserved latanoprost (Xalatan ${ }^{\circledR}$ ) has an immediate deleterious impact on the corneal epithelium, which disappears within 24 hours after exposure due to the regenerative power of the corneal epithelium.

\section{Acknowledgments:}

Disclosure Statement

Disclosure of Interest: The authors report no conflict of interest

\section{Funding details:}

:

\section{Geolocation information}

\section{References}

1. Bean GW, Camras CB. Commercially available prostaglandin analogs for the reduction of intraocular pressure: similarities and differences. Surv Ophthalmol. 
2008;53(Suppl 1):S69-84.

2. Noecker R. Effects of common ophthalmic preservatives on ocular health. Adv Ther. 2001;18(5):205-215.

3. Herreras JM, Pastor JC, Calonge M, et al. Ocular surface alteration after long-term treatment with an antiglaucomatous drug. Ophthalmology. 1992;99(7):1082-1088.

4. Baudouin C. Detrimental effect of preservatives in eyedrops: implications for the treatment of glaucoma. Acta Ophthalmol. 2008;86(7):716-726.

5. Hamacher T, Airaksinen J, Saarela V, et al. Efficacy and safety levels of preserved and preservative-free tafluprost are equivalent in patients with glaucoma or ocular hypertension: results from a pharmacodynamics analysis. Acta Ophthalmol Suppl (Oxf). 2008;86:14-19.

6. Sanford M. Preservative-free latanoprost eye drops in patients with primary openangle glaucoma/ocular hypertension. Clin Drug Investig. 2014;34(7):521-528.

7. Pauly A, Roubeix C, Liang $\mathrm{H}$, et al. In vitro and in vivo comparative toxicological study of a new preservative-free latanoprost formulation. Invest Ophthalmol Vis Sci. 2012;53(13):8172-8180.

8. Chetoni P, Burgalassi S, Monti D, et al. Ocular toxicity of some corneal penetration enhancers evaluated by electrophysiology measurements on isolated rabbit corneas. Toxicol In Vitro. 2003;17(4):497-504.

9. Uematsu M, Kumagami T, Kusano M, et al. Acute corneal epithelial change after instillation of benzalkonium chloride evaluated using a newly developed in vivo corneal transepithelial electric resistance measurement method. Ophthalmic Res. 2007;39(6):308-314.

10. Kusano M, Uematsu M, Kumagami T, et al. Evaluation of acute corneal barrier change induced by topically applied preservatives using corneal transepithelial 
electric resistance in vivo. Cornea. 2010;29(1):80-85.

11. Onizuka N, Uematsu M, Kusano M, et al. Influence of different additives and their concentrations on corneal toxicity and antimicrobial effect of benzalkonium chloride. Cornea. 2014;33(5):521-526.

12. Mohamed YH, Uematsu M, Ueki R, et al. Safety of Sodium Hyaluronate Eye Drop with C12-Benzailonium Chloride. Cutan Ocul Toxicol. 2018:1-26. DOI:10.1080/15569527.2018.1543316

13. Uematsu M, Mohamed YH, Onizuka N, et al. Acute corneal toxicity of latanoprost with different preservatives. Cutan Ocul Toxicol. 2016;35(2):120-125.

14. McKee HD, Gupta MS, Ahad MA, et al. First-choice treatment preferences for primary open-angle glaucoma in the United Kingdom. Eye. 2005;19(8):923-924.

15. Cracknell KP, Grierson I. Prostaglandin analogues in the anterior eye: their pressure lowering action and side effects. Exp Eye Res. 2009;88(4):786-791.

16. Parrish RK, Palmberg P, Sheu WP; XLT Study Group. A comparison of latanoprost, bimatoprost, and travoprost in patients with elevated intraocular pressure: a 12-week, randomized, masked-evaluator multicenter study. Am J Ophthalmol. 2003;135(5):688-703.

17. Crichton AC, Vold S, Williams JM, et al. Ocular surface tolerability in patients with glaucoma or ocular hypertension. Adv Ther. 2013;30(3):260-270.

18. Baudouin C, Riancho L, Warnet JM, et al. In vitro studies of antiglaucomatous prostaglandin analogs: travoprost with and without benzalkonium chloride and preserved latanoprost. Invest Ophthalmol Vis Sci. 2007;48(9):4123-4128.

19. Baudouin C, Labbé A, Liang H, et al. Preservatives in eyedrops: the good, the bad and the ugly. Prog Retin Eye Res. 2010;29(4):312-334.

20. Nakashima M, Nakamura T, Teshima M, et al. Breakdown evaluation of corneal 
epithelial barrier caused by antiallergic eyedrops using an electrophysiologic method. J Ocul Pharmacol Ther. 2008;24(1):43-51.

21. Rouland JF, Traverso CE, Stalmans I, et al. Efficacy and safety of preservative-free latanoprost eyedrops, compared with BAK-preserved latanoprost in patients with ocular hypertension or glaucoma. Br J Ophthalmol. 2013;97(2):196-200.

22. Aptel F, Choudhry R, Stalmans I. Preservative-free versus preserved latanoprost eye drops in patients with open-angle glaucoma or ocular hypertension. Curr Med Res Opin. 2016;32(8):1457-1463.

23. Liang H, Pauly A, Riancho L, Baudouin C, et al. Toxicological evaluation of preservative-containing and preservative free topical prostaglandin analogs on a three dimensional reconstituted corneal epithelium system. $\mathrm{Br} \quad \mathrm{J}$ Ophthalmol. 2011;95(6):869-875.

24. Pellinen P, Huhtala A, Tolonen A, et al. The cytotoxic effects of preserved and preservative-free prostaglandin analogs on human corneal and conjunctival epithelium in vitro and the distribution of benzalkonium chloride homologs in ocular surface tissues in vivo. Curr Eye Res. 2012;37(2):145-154.

25. Martone G, Frezzotti P, Tosi GM, et al. An in vivo confocal microscopy analysis of effects of topical antiglaucoma therapy with preservative on corneal innervation and morphology. Am J Ophthalmol. 2009;147(4):725-735.

26. Kim MS, Choi CY, Kim JM, et al. Microbial contamination of multiply used preservative-free artificial tears packed in reclosable containers. Br J Ophthalmol. 2008;92(11):1518-1521.

27. Majumdar S, Hippalgaonkar K, Repka MA. Effect of chitosan, benzalkonium chloride and ethylenediaminetetraacetic acid on permeation of acyclovir across isolated rabbit cornea. Int J Pharm. 2008;348(1-2):175-178. 
28. Okabe K, Kimura H, Okabe J, et al. Effect of benzalkonium chloride on transscleral drug delivery. Invest Ophthalmol Vis Sci. 2005;46(2):703-708.

29. Gross RL, Peace JH, Smith SE, et al. Duration of IOP reduction with travoprost BAKfree solution. J Glaucoma. 2008;17(3):217-222.

30. Pellinen P, Lokkila J. Corneal penetration into rabbit aqueous humor is comparable between preserved and preservative-free tafluprost. Ophthalmic Res. 2009;41(2):118-122.

31. Baudouin C, Hamard P, Liang H, et al. Conjunctival epithelial cell expression of interleukins and inflammatory markers in glaucoma patients treated over the long term. Ophthalmology. 2004;111(12):2186-2192. 


\section{Figure captions}

Figure 1. Relative corneal TER after instillation of PF latanoprost (Monoprost ${ }^{\circledR}$ ) and preserved latanoprost $\left(\right.$ Xalatan $\left.{ }^{\circledR}\right)$ at different time periods. Data represent the percentage compared to the HBSS control. The values for the preserved group were significantly lower than the other groups only at 1 minute. ${ }^{*} \mathrm{p}<0.01$ (Tukey test, versus control, preserved 1 day group, and preserved 1 week group). $\uparrow \mathrm{p}<0.01$ (Student's t-test, versus PF 1 minute group).

TER, transepithelial electrical resistance; HBSS, Hank's balanced salt solution.

Figure 2. SEM images of the corneal epithelium after instillation of HBSS (control). (A) 1 minute, (B) 1 day, (C) after PF latanoprost (Monoprost $\left.{ }^{\circledR}\right)$ instillation, and (D) 24 hours after 1 week of daily administration of PF latanoprost (12000× magnification). All images show that the corneal epithelial structures were almost entirely intact.

SEM, scanning electron microscopy; HBSS, Hank's balanced salt solution.

Figure 3. SEM images of the corneal epithelium at 1 minute (A) and 1 day (B) after instillation of preserved latanoprost (Xalatan $\left.{ }^{\circledR}\right)$, and 24 hours after 1 week of daily administration of preserved latanoprost (C) (12000× magnification). Figure A shows that the superficial cells of the corneal epithelium were damaged, exhibited lost or degenerated microvilli, lost their adhesions with adjacent cells, and cracks appeared between the cells. Figure parts B and C show that the corneal epithelial structures were almost intact.

SEM, scanning electron microscopy 
Figure 1

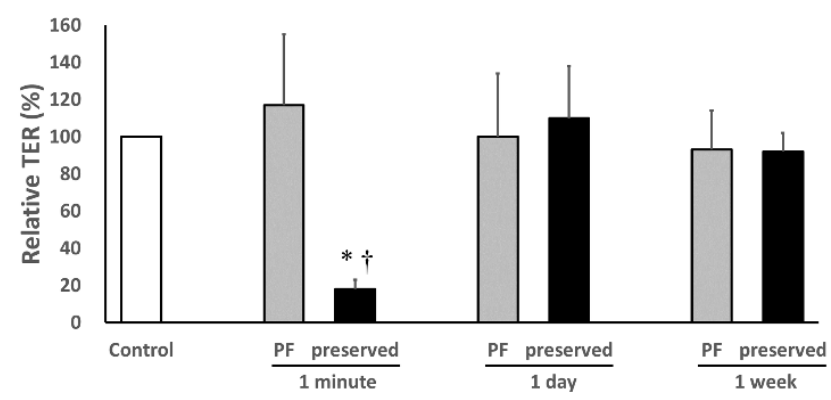


Figure 2
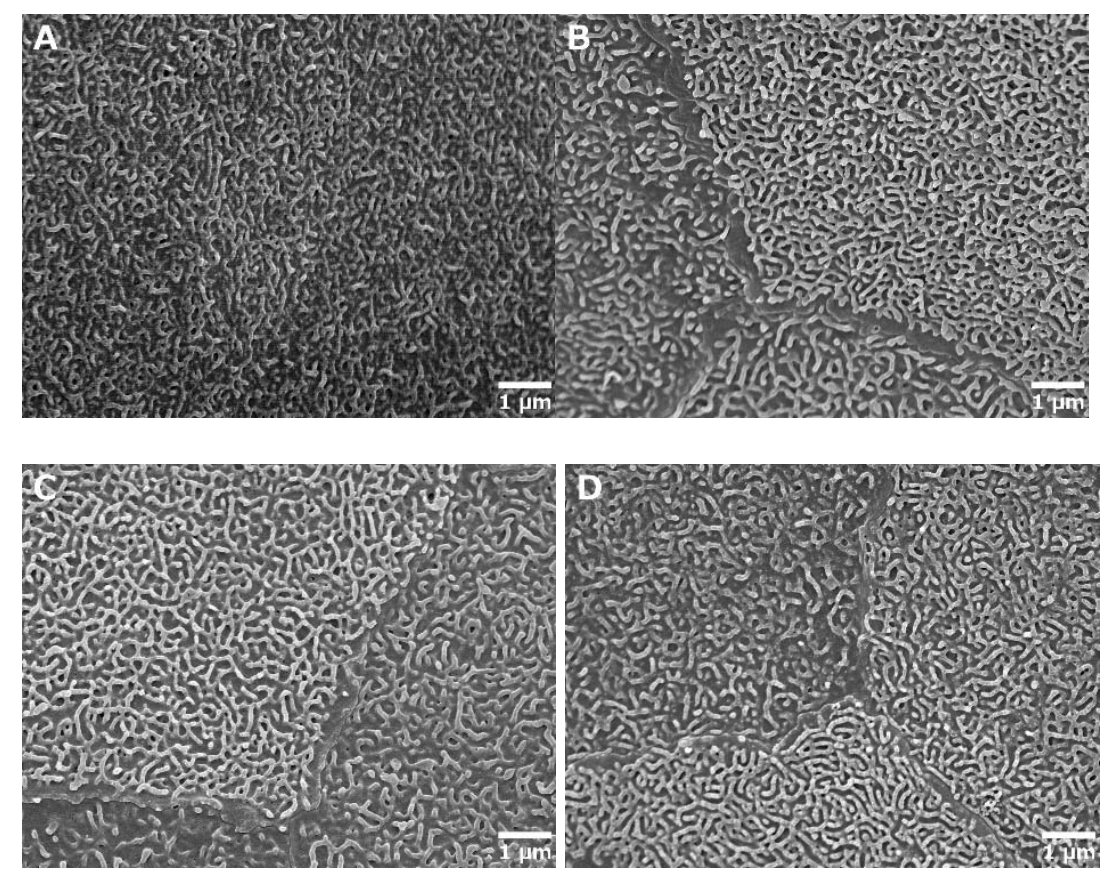
Figure 3
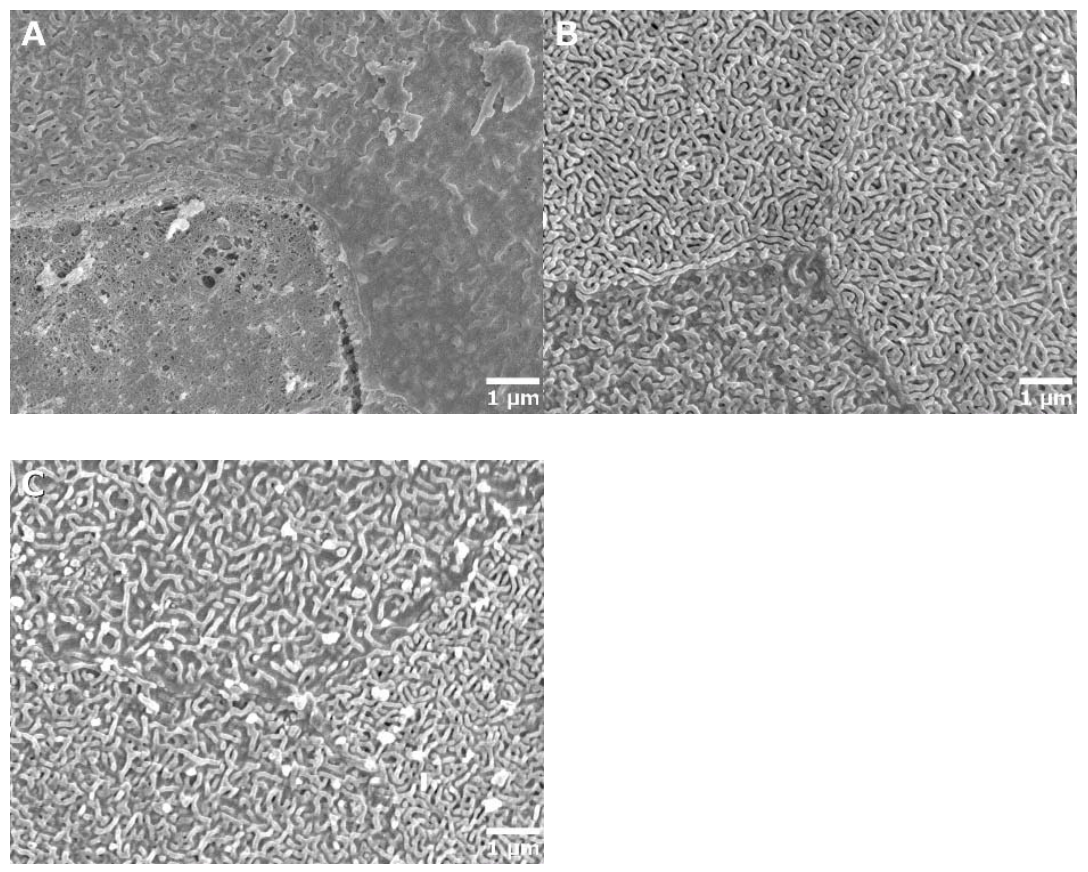